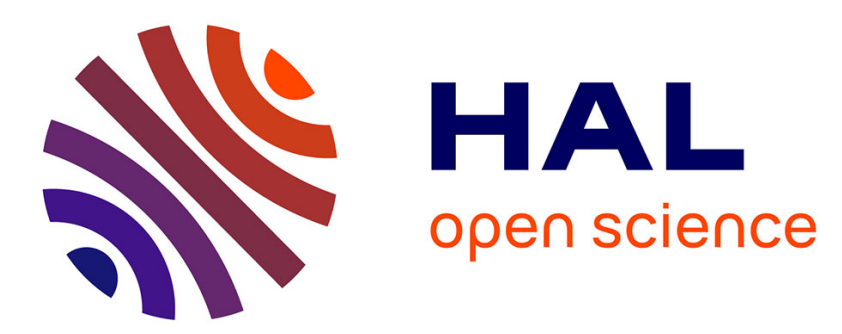

\title{
Warming tolerance across insect ontogeny: influence of joint shifts in microclimates and thermal limits
}

\author{
Sylvain Pincebourde, Jérome Casas
}

\section{To cite this version:}

Sylvain Pincebourde, Jérome Casas. Warming tolerance across insect ontogeny: influence of joint shifts in microclimates and thermal limits. Ecology, 2015, 96, pp.986 - 997. 10.1890/14-0744.1.sm . hal-01395126

\author{
HAL Id: hal-01395126 \\ https://hal.science/hal-01395126
}

Submitted on 10 Nov 2016

HAL is a multi-disciplinary open access archive for the deposit and dissemination of scientific research documents, whether they are published or not. The documents may come from teaching and research institutions in France or abroad, or from public or private research centers.
L'archive ouverte pluridisciplinaire HAL, est destinée au dépôt et à la diffusion de documents scientifiques de niveau recherche, publiés ou non, émanant des établissements d'enseignement et de recherche français ou étrangers, des laboratoires publics ou privés. 


\title{
Warming tolerance across insect ontogeny: influence of joint shifts in microclimates and thermal limits
}

\author{
Sylvain Pincebourde ${ }^{1}$ And Jérôme Casas \\ Institut de Recherche sur la Biologie de l'Insecte (IRBI), CNRS UMR 7261, Université Francois Rabelais, \\ Faculté des Sciences et Techniques, 37200 Tours, France
}

\begin{abstract}
The impact of warming on the persistence and distribution of ectotherms is often forecasted from their warming tolerance, inferred as the difference between their upper thermal limit and macroclimate temperature. Ectotherms, however, are thermally adapted to their microclimates, which can deviate substantially from macroscale conditions. Ignoring microclimates can therefore bias estimates of warming tolerance. We compared warming tolerance of an insect across its ontogeny when calculated from macro- and microclimate temperatures. We used a heat balance model to predict experienced microclimate temperatures from macroclimate, and we measured thermal limits for several life stages. The model shows a concomitant increase in microclimate temperatures and thermal limits across insect ontogeny, despite the fact that they all share the same macroclimate. Consequently, warming tolerance, as estimated from microclimate temperature, remained constant across ontogeny. When calculated from macroclimate temperature, however, warming tolerance was overestimated by $7-10^{\circ} \mathrm{C}$, depending on the life stage. Therefore, errors are expected when predicting persistence and distribution shifts of ectotherms in changing climates using macroclimate rather than microclimate.

Key words: biophysical ecology; ectotherms; global warming; heat tolerance; herbivores; insect ontogeny; leaf temperature; life cycle; microclimate; Phyllonorycter blancardella; upper thermal limit; warming tolerance.
\end{abstract}

\section{INTRODUCTION}

Not all species will experience global warming equally. First, organisms live in microclimates, the temperatures of which can deviate substantially from macroclimate temperatures (Gates 1980, Angilletta 2009). Second, the physiological performance and tolerance of organisms filters microclimate conditions, often nonlinearly (Huey and Kingsolver 1993, Huey et al. 2012). Consequently, the impact of warming on the persistence and distribution of species cannot be inferred solely from macroclimatic observations. Instead, what is required is a mechanistic understanding of both microclimate and the physiological tolerance of species (Williams et al. 2008, Helmuth et al. 2010). For instance, tropical ectotherms were predicted to suffer the most from global warming because they already live close to their thermal limits, whereas temperate ectotherms would benefit from a higher warming tolerance: a larger difference between macroclimate temperature and thermal limits (Deutsch et al. 2008, Tewksbury et al. 2008). Recently, a study showed that equilibrium body temperatures of most ectotherms at all latitudes are higher than both their thermal limits and ambient air

Manuscript received 22 April 2014; revised 11 September 2014; accepted 17 September 2014. Corresponding Editor: T. D. Williams.

${ }^{1}$ E-mail: sylvain.pincebourde@univ-tours.fr temperature (inferred as the highest monthly mean of daily maximum air temperature), suggesting the need to thermoregulate for the maintenance of populations (Sunday et al. 2014). However, the importance of microclimates is often neglected in studies on warming tolerance of ectotherms, as we will detail (Potter et al. 2013, Scheffers et al. 2013a).

Warming tolerance (WT) is calculated as the deviation between the thermal limit-e.g., the critical maximal temperature, CTmax, or the upper lethal temperature, ULT - and the habitat temperature, which usually is taken as a monthly or annual average of macroscale air temperature (Deutsch et al. 2008). On that basis, a direct relationship between warming tolerance and absolute latitude is expected in ectotherms because macroclimate temperature increases from the poles to the equator and there is no clear pattern of CTmax or ULT over latitudes (Addo-Bediako et al. 2000, Deutsch et al. 2008, Sunday et al. 2011, Diamond et al. 2012, Araújo et al. 2013). Frequently, this latitudinal pattern of warming tolerance is used to forecast potential distribution shifts in ectotherms caused by global warming (Sunday et al. 2012). Macroclimate temperature, however, can be uninformative regarding the actual relationship between physiological tolerance and latitude, because the pertinent variable often is the microclimate temperature (Fig. 1; see Huey et al. 2012, Sunday et al. 2014). Two lines of evidence suggest that lack of knowledge on microclimates 
prevents a better understanding of global patterns in thermal limits. First, studies on Drosophila show that the physiological limit correlates with the temperature actually experienced by the insect (Hoffmann et al. 2013). Second, microclimate variability is known to drive thermal adaptations at the local scale along sharp environmental gradients in rocky intertidal zones (Stillman and Somero 2000) and in tropical forests (Scheffers et al. 2013a,b).

Microclimate can be highly heterogeneous in space due to the interactive influences of abiotic variables and physical characteristics of a given habitat, such as topography, nature of the substratum, and architecture of the elements composing the habitat (Gates 1980, Helmuth 1998, Kearney et al. 2010b). Deviations of microclimates from macroscale conditions seem to be the rule rather than the exception (e.g., Gates 1980, Kearney et al. 2014). Fortunately, microclimate temperature can be predicted using mechanistic biophysical models. These mechanistic models operate at the individual level, by translating macroclimate temperature into microclimate temperature and further into performance metrics, or by calculating a threshold for persistence from thermal limits. They operate at the population level, too, by incorporating life histories or energetics to predict population dynamics (Buckley et al. 2010). Biophysical ecology proposes the most integrative approach by modeling species niches using physical laws for heat transfer between the organism's body and its environment (Gates 1980), and by incorporating physiological traits such as thermal limits and performance relationships (Fig. 1; see also Kearney et al. 2010b). The coupling of a biophysical model and physiological limits was shown to be successful at predicting microclimatic or body temperature patterns from climatic data and inferring thermal stress or potentially lethal exposure in various systems (Sunday et al. 2014), including crustaceans in the intertidal zone (Helmuth et al. 2006, Denny et al. 2009), lizards (Kearney 2013), insects (Pincebourde et al. 2007, Kearney et al. 2010a), and mammals (Natori and Porter 2007). Here, we applied this biophysical modeling approach to an insect-plant relationship to gain insight into the link between the thermal limits and the microclimate temperatures across the life cycle of an insect. Thermal limits can vary for each life stage in ectotherms, but these are rarely integrated into biophysical frameworks of climate change impacts (Kingsolver et al. 2011, Briscoe et al. 2012). This comprehensive approach is necessary, however, because the most sensitive life stage will be the one defining the range of sustainable variations for the species.

We compared the thermal limits and the microclimate temperatures across the life cycle of the spotted tentiform leaf miner, Phyllonorycter blancardella (see Plate 1), to (1) identify the most and the least tolerant life stages, and (2) to test the hypothesis that each stage is thermally adapted to its microenvironment: warming tolerance should then remain constant across insect

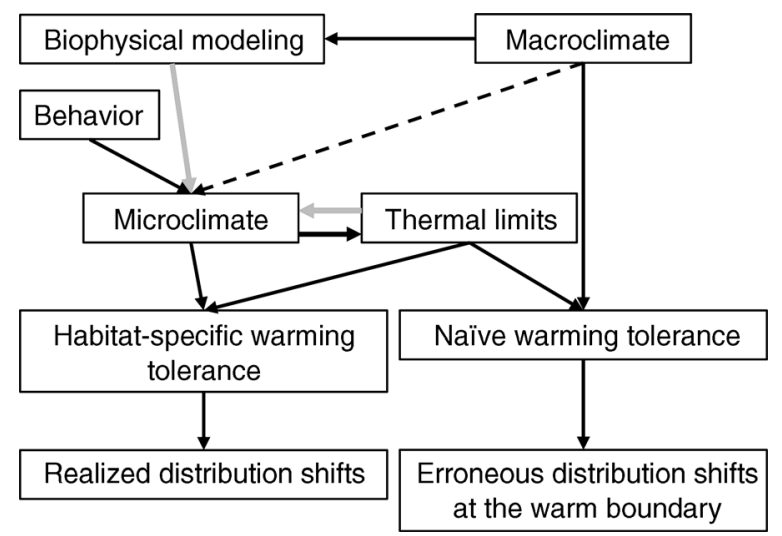

FIG. 1. Conceptual framework for studying warming tolerance of ectotherms. Because of the lack of knowledge about the microclimate experienced by most organisms, warming tolerance is usually inferred from macroclimate temperatures; warming tolerance is then called "naïve" (Scheffers et al. 2013a). This naïve approach can lead to erroneous forecasts of distribution shifts in species range, which are overestimated or underestimated if the microhabitat amplifies or buffers maximal environmental temperatures, respectively. In contrast, using microclimate temperature and knowledge about behavioral thermoregulation strategies, one can predict a "habitat-specific" warming tolerance and hence predict a realistic distribution shift. The gray arrows indicate the inverse approach, allowing one to estimate the microclimate variables from thermal limits (see Discussion).

development, despite varying thermal limits. The larva of this ecosystem engineer develops within apple leaf tissues, inside a gallery called a mine. This species is nearly sessile (i.e., a larva completes development inside a unique mine), meaning that there is little opportunity for behavioral thermoregulation to influence thermal responses. The moth experiences microclimatic warming by the end of its larval development as the mine reaches temperatures up to $10^{\circ} \mathrm{C}$ above ambient when in full sunlight (Pincebourde and Casas 2006a, Pincebourde et al. 2007). This phenomenon is caused by feeding behavior, which both increases the portion of infrared radiation absorbed by the mine and induces stomatal closure, in particular during the two last larval stages. Indeed, among the five larval stages, the first three larval stages (L1-3) are sap-feeders and the damage that they cause to leaf tissues is barely visible. For this reason, the early larval stages (L1-3) were considered as equivalent to the "egg stage" in this study. Therefore, we predict that the microclimatic change increases in amplitude between the two groups, one consisting of eggs and larvae L1-L3, and the other consisting of the older larval stages (L4-5) and pupae. We further hypothesized that the thermal limit (ULT) of each stage follows this microclimate trend. Thus, we compared a "habitatspecific" warming tolerance, defined as the difference between microclimate temperature and ULT, to a "naïve" warming tolerance as derived from macroclimate temperature and ULT (Fig. 1; see Scheffers et al. 2013a). Our approach was based on the development of 


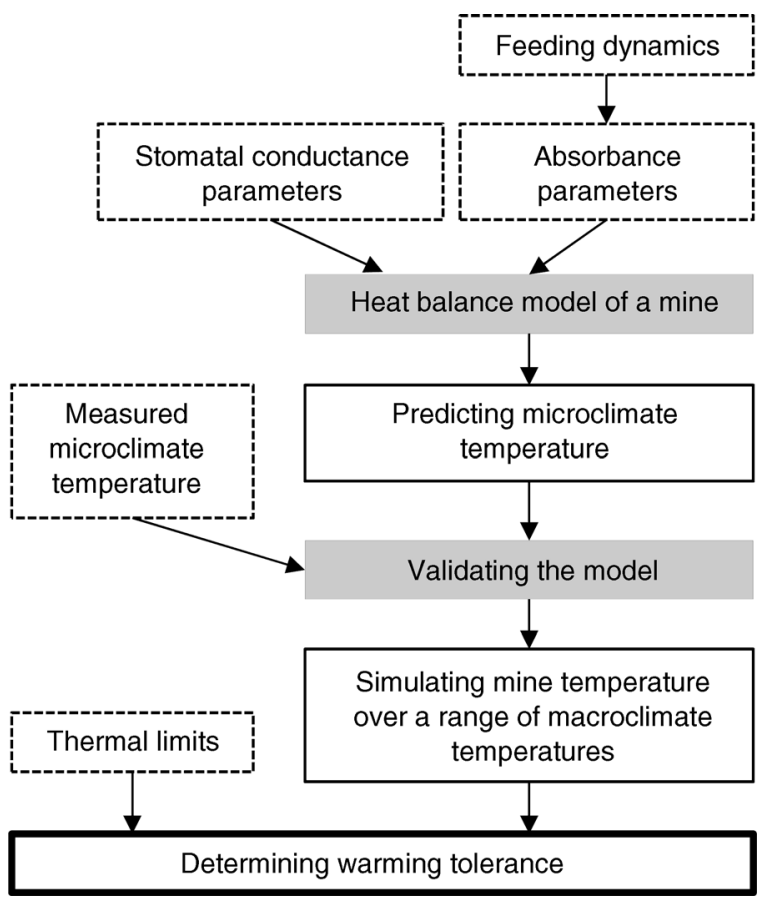

FIG. 2. Workflow of the necessary steps leading to the estimation of warming tolerance for different life stages. Dashed boxes represent experimental measurements, gray boxes concern the model, and solid-outlined boxes are for model outputs. The model was parameterized using measurements of maximal stomatal conductance and feeding dynamics. Feeding dynamics measurements were necessary to estimate absorbance values. The heat balance model was tested by comparing predictions and microclimate temperature measurements. The validated model was run to simulate microclimate temperature to determine further warming tolerances over a wide range of macroclimate temperatures, in combination with measurements of thermal limits. This entire sequence of actions was repeated for the different life cycle stages of the spotted tentiform leaf miner, Phyllonorycter blancardella: egg, larvae (L4, L5), and pupae.

a heat budget model to predict the temperature within a mine from the macroclimate, the stomatal behavior, and the damage caused by the feeding activity of the larvae (Fig. 2 displays the different steps of our study). We first parameterized the model with measurements of stomatal conductance and feeding activity. Then we validated the model by comparing predictions and experimental measurement of mine temperatures for each life stage (egg, L4, L5, and pupae). Finally, we combined thermal limit measurements and model simulations to calculate warming tolerance for each life stage across a range of macroclimate temperatures.

\section{Methods}

\section{The plant and insect species}

The life cycle of the spotted tentiform leaf miner Phyllonorycter blancardella (Fabricius 1781) includes the egg, five larval stages, the pupae, and the adult (see Plate 1). The adult lays eggs individually at the lower surface of apple leaves. The first-stage larva (L1) penetrates leaf tissues. The first three larval stages (L1-3) are sapfeeders. The L3 outlines the mine within which the two last larval stages (L4 and L5) and the pupae will further develop. L4 and L5 are tissue-feeders. This feeding behavior results in the formation of translucent spots at the upper leaf surface, called feeding windows (Djemai et al. 2000). Feeding windows are adjacent to green patches, which correspond to the chlorophyll-containing tissues that have not been consumed by the larva. Leaf mines are small structures of $\sim 1 \mathrm{~cm}^{2}$, although with a large volume relative to L5 body size due to the tentiform shape of the mine (Pincebourde et al. 2006). Phyllonorycter blancardella was reared on 1-3 year-old apple seedlings (Malus domestica, Golden cultivar) in a greenhouse in Tours, France $\left(47^{\circ} 21^{\prime} \mathrm{N}, 0^{\circ} 42^{\prime} \mathrm{E}\right)$. Initially, populations were collected in the vicinity of Tours in 2003 and maintained for several years. In the greenhouse, the daily amplitude of change of conditions was similar from day to day during spring, in particular daily air temperature (from minimum to maximum $14.5^{\circ}$ to $38.5^{\circ} \mathrm{C}$, mean $24.4^{\circ} \mathrm{C}$ ), daily relative humidity $(29.5-$ $95 \%$, mean $74 \%$ ), and radiation load, which was up to $875 \mathrm{~W} / \mathrm{m}^{2}$. Insects from spring generations were used in all experiments. Apple seedlings were planted in earthenware pots $(11.5 \mathrm{~cm}$ in diameter) and watered every two days. A nutritive solution was added to the water once per month (volume fractions: $6 \%$ nitrogen, $6 \% \mathrm{P}_{2} \mathrm{O}_{5}$, and $6 \% \mathrm{~K}_{2} \mathrm{O}$ ).

\section{The biophysical model of microclimate temperatures}

We used a biophysical model to understand the mechanisms underlying the microclimatic temperature patterns over the course of leaf miner ontogeny. The heat budget of a L5 mine was first developed by Pincebourde and Casas (2006a). It was tested and validated under both fully controlled experimental conditions and field conditions (Pincebourde et al. 2007). Briefly, this model computes heat transfer between a L5 mine and its surroundings, including (1) radiation absorption and emission, (2) convective heat loss, (3) conduction of heat between a mine and the adjacent intact leaf tissues, and (4) latent heat loss during evapotranspiration via stomata. The model first computes the temperature of intact leaf tissues adjacent to a mine (Campbell and Norman 1998, Nobel 1999), and then the temperature within a mine according to numerous parameters belonging to leaf and mine allometries, stomatal conductance, absorbance values, and environmental variables (Pincebourde and Casas 2006a). The parameters that vary during ontogeny concern the radiative energy and the latent heat terms. The latter is based on the Jarvis (1976) submodel for stomatal conductance (see Appendix: Eqs. A.1-3). Feeding behavior alters the optical properties of leaf tissues, and hence absorbance values vary (Pincebourde and Casas 2006a, b). Also, stomata in the mine integument respond differently to environmental 

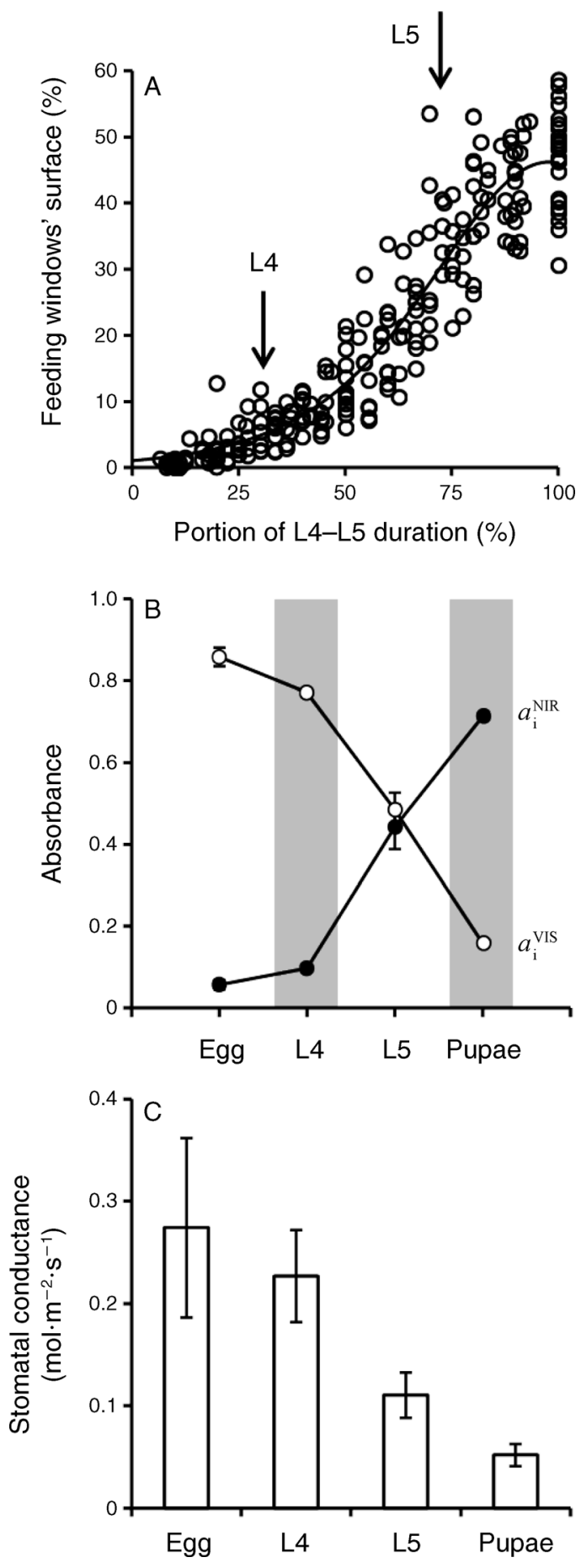

FIG. 3. (A) Dynamics of the feeding windows' surface area as a percentage of total mine surface area across the development of L4 and L5 larval stages, where developmental time is expressed as a percentage of the total time needed to achieve the L4-L5 development. The nonlinear fit is the logistic regression model. Arrows indicate the approximate location of the middle of the L4 and L5 stages. (B) The dynamics of mine surface absorbance in the visible ( $a_{\mathrm{i}}^{\text {VIS }}$; open circles) and near infrared ( $a_{\mathrm{i}}^{\mathrm{NIR}}$; solid circles) across life stages; absorbance values taken from Pincebourde and Casas (2006a) were obtained using a spherical spectrometer. Measured values are indicated as changes (Pincebourde et al. 2006). The parameters for the other heat budget terms were given elsewhere (Pincebourde and Casas 2006a). The biophysical model was thus modified to compute the microclimatic temperature of L4 mines and pupae. For eggs, we considered that their microclimatic temperature matches the temperature of intact leaf tissues. This assumption was later validated by direct temperature measurements. We also considered that the microclimates of stages L1, L2, and L3 were similar to that of intact leaf tissues, and therefore to eggs, because at these stages (1) the mine is not tentiform, (2) there are no feeding windows, and (3) the mine is barely visible from the lower leaf surface.

\section{Feeding dynamics during insect ontogeny}

Eggs and stages L1 to L3 do not make feeding windows. Feeding behavior was recorded along the development of L4 and L5 to test if it generates a shift in microclimate temperature across ontogeny. We used feeding dynamics further to parameterize the heat budget model. Feeding was quantified by measuring the rate of appearance of feeding windows at the mine surface. Feeding dynamics were followed on 23 mines from eight different apple seedlings. The mines were held under greenhouse conditions throughout the experiment, with a mean ambient temperature of $24.4^{\circ} \mathrm{C}$ (see The plant and insect species). At the same time every day, a digital picture of the upper mine surface was taken under a binocular (zoom $5 \times$ ). Pictures were analyzed in Scion Image software (Scion, Frederick, Maryland, USA) to measure the total mine surface area and the portion of feeding windows every day. All plants were grown in the greenhouse throughout the experiment, which ended when the surface area of feeding windows remained constant, suggesting that pupation had started. The surface area of feeding windows was expressed as a function of the portion of development time, which corresponds to the total duration of L4-L5 development. A logistic regression analysis was performed to describe the temporal dynamics of the portion of feeding windows using TableCurve software version 5.01 (SYSTAT 2002, Chicago, Illinois, USA). The inflexion point of the logistic model was used to distinguish between the two stages L4 and L5 (Fig. 3A). From this relationship, we inferred that the middle of stages L4 and L5 occurred at $30 \%$ and $70 \%$ of total development time, respectively.

\section{Dynamics of absorbance during insect ontogeny}

To parameterize the biophysical model, absorbance values were estimated for all stages. Absorbance in the

mean $\pm \mathrm{SD}$; values in the gray bands are estimates. (C) Dynamics of maximal stomatal conductance in the mine integument (mean $\pm \mathrm{SD})$ across life stages. 
visible $\left(a_{\mathrm{i}}^{\mathrm{VIS}}\right)$ and near-infrared $\left(a_{\mathrm{i}}^{\mathrm{NIR}}\right)$ ranges of the solar spectrum are known for intact apple leaf tissues and L5 mines (Pincebourde and Casas 2006a). These values, combined with the temporal dynamics of window formation, were used to infer absorbance values at the L4 and pupae stages (see Appendix). Our method assumes that the decrease in absorbance in the visible range is proportional to the decrease in the portion of green patches remaining in a mine, whereas the increase in absorbance in the near-infrared range correlates with the increase in the portion of feeding windows.

\section{Dynamics of stomatal conductance during insect ontogeny}

The latent heat term of the biophysical model was parameterized by measuring the maximal stomatal conductance $\left(g_{i}^{\text {smax }}\right)$ at egg stage (intact leaf tissues), and L4, L5, and pupae mines. Gas exchange was measured with an infrared gas analyzer equipped with a $2 \times 3 \mathrm{~cm}$ leaf chamber system (LI-6400, LI-COR, Lincoln, Nebraska, USA) and with an external light source (6400-02B, LI-COR) following the method given in Pincebourde et al. (2006); see Appendix. Leaves were cut and scanned after measurements. Scans were analyzed using ImageJ software version 1.47 (available online $)^{2}$ to measure mine areas in order to express stomatal conductance per unit of transpiring surface.

\section{Microclimate temperature measurements for model validation}

To test model predictions, we measured the microclimate temperature for eggs, L4, L5, and pupae. Environmental conditions during measurements were typical for mines exposed to solar radiation in spring in France (Pincebourde et al. 2007): i.e., air temperature $25^{\circ} \mathrm{C}$, relative humidity $65 \%$, radiation level at leaf surfaces ranging between 516 and $565 \mathrm{~W} / \mathrm{m}^{2}$, and wind velocity $0.3 \mathrm{~m} / \mathrm{s}$. Microclimatic temperatures were recorded by placing apple seedlings containing leaf mines in a Conviron chamber (VB 1014-A, Vötsch, Balingen Frommern, Germany) which allowed full control of the environment. Two metal halide lamps ( $250 \mathrm{~W}$; Sylvania Britelux HSI-T SX clear) mimicked the solar spectrum. We measured microclimate temperatures using fine thermocouples as in Pincebourde and Casas (2006a); see Appendix. The chamber setup allowed us to follow the temperature of, at best, four leaves or mines at the same time. The same procedure was followed each time: apple seedlings were put in the chamber under the conditions mentioned previously, and thermocouples were positioned. After an acclimation period of $1.5 \mathrm{~h}$, microclimatic temperatures were sampled every minute for 30 minutes, and we calculated an average over this period of the assay. We recorded microclimatic temperatures for 10 eggs, 10 L4 mines, 13 L5 mines, and 10 pupae. We tested the validity of the

2 http://imagej.nih.gov/ij/ biophysical model for each leaf miner stage by comparing the microclimatic temperature measurements to the predictions of the model. We calculated the root mean square of error of predictions (RMSEP) as a quantitative estimate of model accuracy.

\section{Upper lethal temperatures}

We measured the upper lethal temperature (ULT) of eggs, L4, L5, and pupae. ULT is defined here as the temperature causing a mortality rate of $50 \%$ in a given group (lethal dose, LD50) after exposure for $1 \mathrm{~h}$. Both experimental and modeling studies showed that the daily maximal temperature experienced by the leaf miner lasts for about $1 \mathrm{~h}$ (Pincebourde et al. 2007, Saudreau et al. 2013). A ramping temperature assay was applied to simulate realistic thermal conditions (see Discussion, on the influence of methodology on thermal limit determination). Field measurements showed that the temperature in mines exposed to solar radiation can change at a rate of $0.37^{\circ} \mathrm{C} / \mathrm{min}$, on average, with a range of $0.11-$ $3.35^{\circ} \mathrm{C} / \mathrm{min}$ (Pincebourde et al. 2007). This fast rate of temperature increase arises when a mine is suddenly exposed to solar radiation after being in the shade or under cloudy conditions. The experiments were completed within the same week in spring 2004, and all insects experienced very similar daily temperature conditions in the greenhouse during the two weeks prior to the tests (i.e., mean daily air temperature $25.3^{\circ} \mathrm{C}$, mean daily minimum air temperature $18.1^{\circ} \mathrm{C}$, and mean daily maximum air temperature $34.8^{\circ} \mathrm{C}$ ). The procedure was to put a group of 6-13 individuals of a given stage within the climatic chamber (VB 1014-A, Vötsch) to measure mortality after $1 \mathrm{~h}$ of exposure to a given experimental temperature. Insects were extracted from their mines in the morning, when air temperature was around $25^{\circ} \mathrm{C}$, and were put immediately into a petri dish just before the experiment started. The petri dish was equipped with a fine copper-constantan thermocouple (type T, $0.2 \mathrm{~mm}$ in diameter; TCSA, Dardilly, France) measuring the temperature inside the dish close to the insects. A wet piece of cotton generated high humidity within the sealed petri dish such that insects did not desiccate. After an acclimation period of $15 \mathrm{~min}$ at $29^{\circ} \mathrm{C}$, the temperature was ramped up at $0.32^{\circ} \mathrm{C} / \mathrm{min}$ until reaching the experimental temperature, which was maintained for $1 \mathrm{~h}$. Survival was assessed $15 \mathrm{~h}$ after the treatment; during that time, insects were conserved within the petri dishes at $25^{\circ} \mathrm{C}$. The test consisted of touching larvae and pupae with a fine needle. If alive, they respond by moving violently, which is a defense against parasitoids (Djemai et al. 2000). Each group was exposed to a single temperature treatment. Overall, 461 individuals in total were processed. For each life stage, nonlinear regression was used to estimate ULT. We fitted a logistic dose response model using the software TableCurve (SYSTAT), composed of four parameters including the LD50 value (Pincebourde et al. 2007, 2008). 


\section{Model exploration}

The biophysical model was used to reveal the mechanisms underlying the shift in microclimate temperature across leaf miner ontogeny. This exploration focused on the impact of varying radiation level, because it is the only environmental variable shared between the equations describing the insect-induced modifications in radiation and latent heat terms. The parameterized biophysical model ran for each leaf miner stage from radiation level 0 to $900 \mathrm{~W} / \mathrm{m}^{2}$. The other environmental variables were kept constant (macroclimate temperature $25^{\circ} \mathrm{C}$, relative humidity $65 \%$, wind speed $0.3 \mathrm{~m} / \mathrm{s}$ ). The portions of radiation corresponding to the PAR and NIR were kept at 0.37 and 0.63 , respectively (Conviron chamber conditions). Parameters other than those measured here are given in Pincebourde and Casas (2006a). Finally, we back-calculated the net radiation term, to quantify the impact of changes in absorbance on the radiative energy acquired during each leaf miner stage, as well as the latent energy term, to quantify the impact of changes in stomatal behavior on the energy lost during evapotranspiration (see relevant equations in the Appendix).

\section{Predicting warming tolerance across ontogeny}

We used the biophysical model to quantify the impact of macroclimate warming on microclimate temperatures and on warming tolerance of each leaf miner stage. First we computed microclimatic temperatures as a function of macroclimate temperature from $25^{\circ} \mathrm{C}$ to $38^{\circ} \mathrm{C}$ with a $1^{\circ} \mathrm{C}$ increment, while keeping all other variables constant to match natural conditions (relative humidity $65 \%$, radiation level $810 \mathrm{~W} / \mathrm{m}^{2}$, ratio VIS/NIR of 0.82 ). Second, we calculated the warming tolerance of each leaf miner stage at each air temperature increment. A "habitat-specific" warming tolerance was calculated as the difference between microclimate temperature (i.e., temperature within a mine) and ULT for a given stage. We also calculated a "naïve" warming tolerance for each stage, based this time on macroclimate temperature and not on the mine temperature, to quantify the error of prediction due to neglecting the microclimatic component (Scheffers et al. 2013a). A positive warming tolerance indicates that the insect can sustain a given amplitude of warming before $50 \%$ of the population dies. In contrast, a negative warming tolerance suggests that the tolerance threshold has already been exceeded.

\section{RESUlTS}

\section{Dynamics of feeding, absorbance, and stomatal conductance across ontogeny}

The combined L4 and L5 stages needed 9-12 days to develop, with the exception of a single larva that needed 15 days. The appearance of feeding windows during these stages followed a nonlinear relationship with time (Fig. 3A; logistic regression model: $r^{2}=0.90, F_{2,231}=$

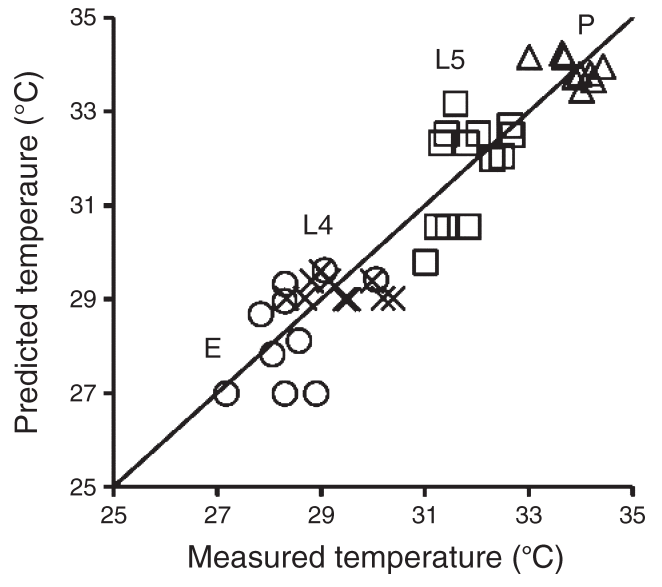

FIG. 4. Validation of the biophysical model by comparing predicted microclimate temperatures to the microclimate temperatures measured experimentally for the life stages: eggs (E; circles), L4 (crosses), L5 (squares), and pupae (P; triangles). The line indicates 1:1 equality.

1028.65, $P<10^{-5}$ ). The surface area of feeding windows $\left(S^{\mathrm{FW}}\right)$ increased slightly during the L4 stage. Then, the rate of feeding window formation increased as the larva entered the L5 stage. The consequence of this nonlinearity was lower absorbance of visible wavelengths, and higher absorbance in the near infrared, as the larva develops (Fig. 3B).

Maximal level of stomatal conductance $\left(g^{\text {smax }}\right)$ decreased over the course of the leaf miner ontogeny (Fig. 3C; ANOVA: $F_{3,60}=57.86, P<0.001$ ). While $g^{\text {smax }}$ in L4 mines and intact leaf tissues was comparable (Tukey HSD multiple comparisons, $P=0.048$ ), the levels dropped in L5 and pupae mines (Tukey HSD multiple comparisons, $P<0.01$ for all comparisons involving L5 and pupae). At the pupae stage, $g^{\text {smax }}$ reached an extremely low mean level of 0.052 $\mathrm{mol} \cdot \mathrm{m}^{-2} \cdot \mathrm{s}^{-1}$.

\section{Validation of the microclimate temperature model}

Overall, the model accurately predicted microclimate temperatures across life stages in the mine temperature range of $27-35^{\circ} \mathrm{C}$ (Fig. 4; $\mathrm{RMSEP}=0.80^{\circ} \mathrm{C}$ ), although this varied slightly between life stages, with the RMSEP ranging from $0.58^{\circ} \mathrm{C}$ for pupae to $0.93^{\circ} \mathrm{C}$ for eggs (Fig. 4). Overall, microclimatic temperatures were always higher than ambient air temperature, and they increased across leaf miner ontogeny, despite identical environmental conditions (see Appendix: Fig. A1).

\section{Microclimatic shift across ontogeny}

The biophysical model predicted an increase in microclimate temperatures with radiation level at the leaf surface for all stages (Fig. 5A). Eggs and L4 larvae were predicted to experience similar microclimatic temperatures up to $7^{\circ} \mathrm{C}$ above ambient air (at $25^{\circ} \mathrm{C}$ ) at high radiation level. In contrast, a much warmer microclimate was predicted for L5 larvae (temperature 

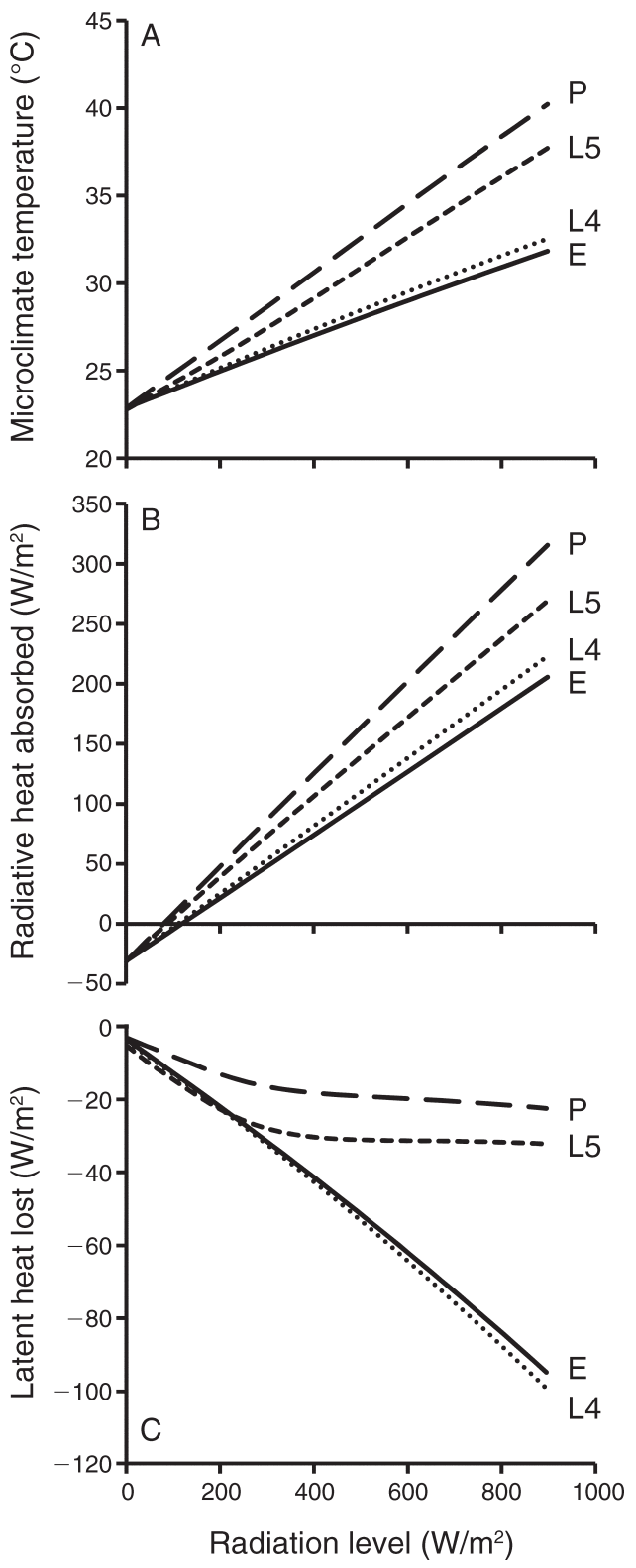

FIG. 5. Microclimate shift as function of radiation level at the leaf surface and at ambient air temperature $25^{\circ} \mathrm{C}$ across the leaf miner life cycle. The biophysical functioning of a mine is driven by the dynamics of (A) microclimate temperature, (B) radiative energy absorbed, and (C) latent heat lost for the different life stages: eggs (E; solid lines), L4 (dotted lines), L5 (dashed lines), and pupae (P; long-dashed lines).

excess up to $12^{\circ} \mathrm{C}$ ) and for pupae (temperature excess up to $15^{\circ} \mathrm{C}$ ). The net radiative energy term showed that the mines of older stages absorb more radiative energy than the younger stages (Fig. 5B). In addition, the latent heat term (the energy lost during evapotranspiration) indicated that the mine of L5 larvae and pupae lost much less energy than the other stages (Fig. 5C) because of the stomatal closure when radiation level increases (see Fig. A2). Increased radiation absorption and lowered evapo- transpiration both contribute to the microclimatic shift during insect ontogeny.

\section{Upper lethal temperature shift across ontogeny}

The thermal limit (ULT) increased across ontogeny (Fig. 6). The logistic dose response regression analysis $\left(P<0.001\right.$ for all; $r^{2}$ ranged from 0.97 to 0.99$)$ positioned the ULT (defined as LD50) at $38.07^{\circ} \mathrm{C}$, $38.35^{\circ} \mathrm{C}, 41.86^{\circ} \mathrm{C}$, and $44.19^{\circ} \mathrm{C}$ for eggs, L4, L5, and pupae, respectively. Therefore, a given microclimate temperature can be lethal for an early stage without causing mortality in a later stage.

\section{Predicting warming tolerance across ontogeny}

Naïve warming tolerance was larger than the habitatspecific warming tolerance by $7-10^{\circ} \mathrm{C}$ (Fig. 7). The naïve warming tolerance was predicted to increase, following the change in thermal limits, across leaf miner life stages. In contrast, our model simulations showed that the habitat-specific warming tolerance was conserved across developmental stages. The slope of the decrease in warming tolerance as ambient air temperature increased was also similar among these stages because the microclimate temperature increases at a similar rate with ambient warming (see Appendix: Fig. A3). All developmental stages (egg, L4, L5, and pupae) were at risk under high-radiation-level at air temperatures of $31-32^{\circ} \mathrm{C}$ and above (Fig. 7).

\section{DisCUSSION}

Thermal limits are available for a large number of species, and for several life stages in some species (AddoBediako et al. 2000, Araújo et al. 2013, Scheffers et al. 2013a). Recently, these data were used to infer the

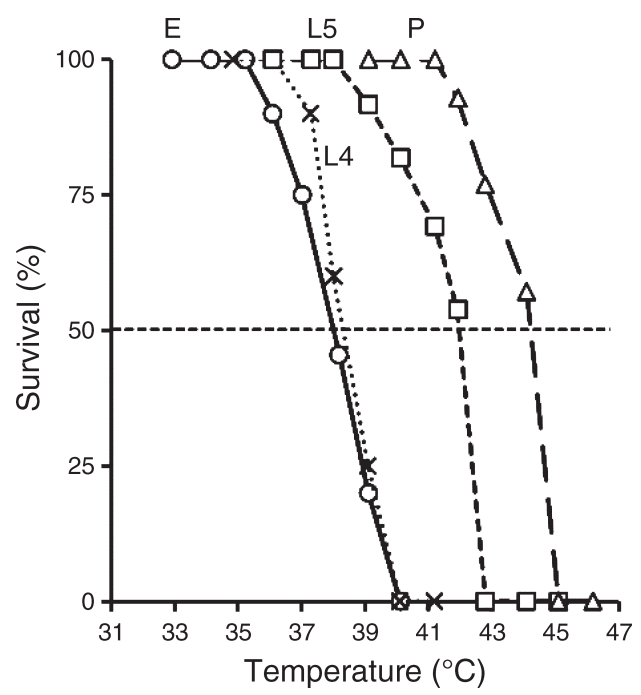

FIG. 6. The shift in thermal limits across ontogeny. Survival rate is shown as function of experienced temperature for each life stage: eggs (E; circles), L4 (crosses), L5 (squares), and pupae ( $\mathrm{P}$; triangles). The horizontal dashed line represents a $50 \%$ survival threshold. 
warming tolerance of ectotherms across latitudes, typically by taking the difference between thermal limits and macroclimate temperatures (Deutsch et al. 2008, Diamond et al. 2012, Sunday et al. 2012, Kingsolver et al. 2013). In $P$. blancardella, the assessment of warming tolerance from the thermal limits alone would suggest that, a priori, the egg is the stage at which the species is the most vulnerable to warming. However, we show that the thermal limits of the leaf miner are tightly linked to the experienced microclimate temperature instead of the macroclimate temperature. Thermal limits and microclimatic temperatures increase concomitantly during ontogeny so that all stages share the same level of tolerance to warming. The mechanistic modeling approach has the advantage of detailing precisely the physical and physiological consequences of warming on microclimatic temperatures actually experienced, and hence the vulnerability of ectotherms to climate change. The model includes two trophic levels (the plant and the insect) across the ontogeny of the insect, an infrequent combination (Pincebourde and Casas 2006a).

Assumptions and simplifications are inherent to any modeling approach. Here, the most important assumption is about the setting of thermal limits, a critical step when estimating warming tolerance (Fig. 2). The methodology is well known to influence the determination of thermal limits (Terblanche et al. 2011, Rezende and Santos 2012). Heating rate and exposure time are among the main factors influencing the thermal limit measurement (Ribeiro et al. 2012, Rezende et al. 2014). We used a heating rate of $0.32^{\circ} \mathrm{C} / \mathrm{min}$ and an exposure time of one hour for several reasons. First, these values are representative of the microclimate temperature patterns measured in the field (Pincebourde et al. 2007). Second, fast rates of temperature increase are often preferred to avoid confounding effects such as starvation (Rezende et al. 2011, Santos et al. 2011). Third, transient exposure in our temperature assays was more realistic than sustained exposure because maximal temperatures within a mine in the field are normally reached around midday, when solar radiation is high and air temperature peaks (Pincebourde et al. 2007). Nevertheless, the upper lethal threshold we measured may not represent an accurate physiological limit to prolonged exposure ( $>1$ hour) to sublethal temperatures, which may happen during heat wave events, for example.

A second assumption is that our model focused on the environmental conditions leading to the highest microclimate temperatures (daily maximum) to compute a standardized warming tolerance for distinct life stages. Thermal history and acclimation, however, are well known to influence physiological limits (Terblanche et al. 2011). Short exposure to high, but nonlethal, temperatures tends to increase subsequent resistance of terrestrial ectotherms to heat, although the upper thermal limits are strongly constrained genetically (Hoffmann et al. 2013). Furthermore, our biophysical

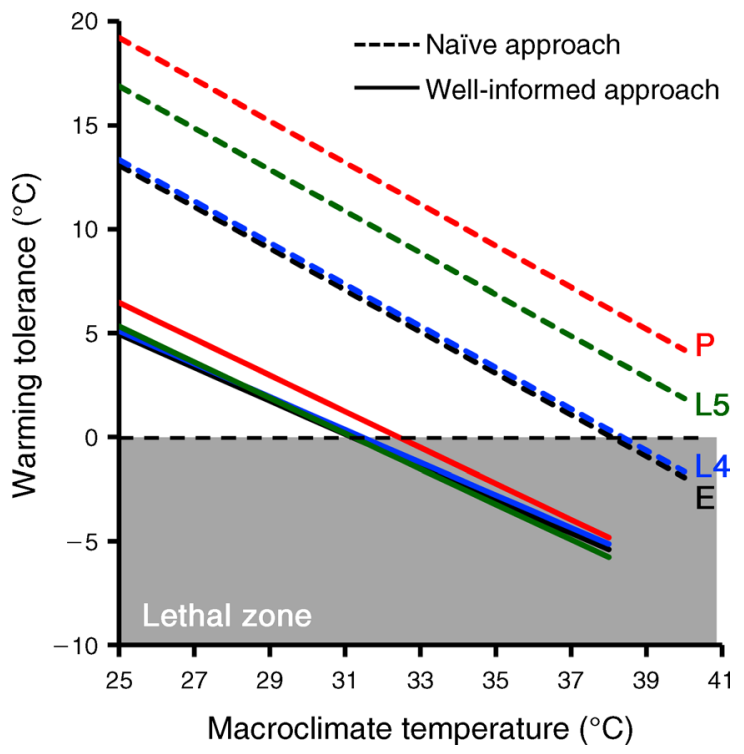

FIG. 7. Habitat-specific, well-informed warming tolerance (upper lethal temperature, ULT, minus microclimate temperature; solid lines) and naïve warming tolerance (ULT minus macroclimate temperature; dashed lines) as function of macroclimate temperature across ontogeny: eggs (E; black lines), L4 (blue lines), L5 (green lines), and pupae (P; red lines). Positive and negative values indicate the ranges of tolerable and critical (gray portion) temperatures, respectively.

model excluded the possibility for phenotypic plasticity in larvae; thermal history at a given life stage may impact the thermal response of the subsequent stages. For example, the temperature experienced by eggs of Manduca sexta alters initial larval growth rate, but it has no persistent effect across all larval stages (Potter et al. 2011). Similarly, the microclimate temperature experienced during larval stages influences the adult phenotype and further survival and/or fitness (Crill et al. 1996, Roberts and Feder 1999). Therefore, the effect of thermal history across life stages in the leaf miner may cause an increase of the upper thermal limit in the older stages (e.g., L5, pupae), leading potentially to an increase in warming tolerance across insect ontogeny. Generally, however, the effect of thermal history and short-term responses to temperature cause variations of a few degrees in the upper thermal limit (e.g., Nyamukondiwa and Terblanche 2010), at best. This remains far below the amplitude of the thermal limit shift that we report across ontogeny of the leaf miner $\left(>7^{\circ} \mathrm{C}\right)$.

Daily maximal microclimate temperature is probably the most direct thermal factor influencing survival, but it does not describe the whole interaction between climate, microclimate, and organisms in itself. Individuals are exposed to daily thermal fluctuations in their habitat. Fluctuating temperatures impact several key parameters, including critical maximal threshold (Paaijmans et al. 2013), metabolic rate and molecular traits (Folguera et al. 2011), developmental time (Kingsolver et al. 2009), 


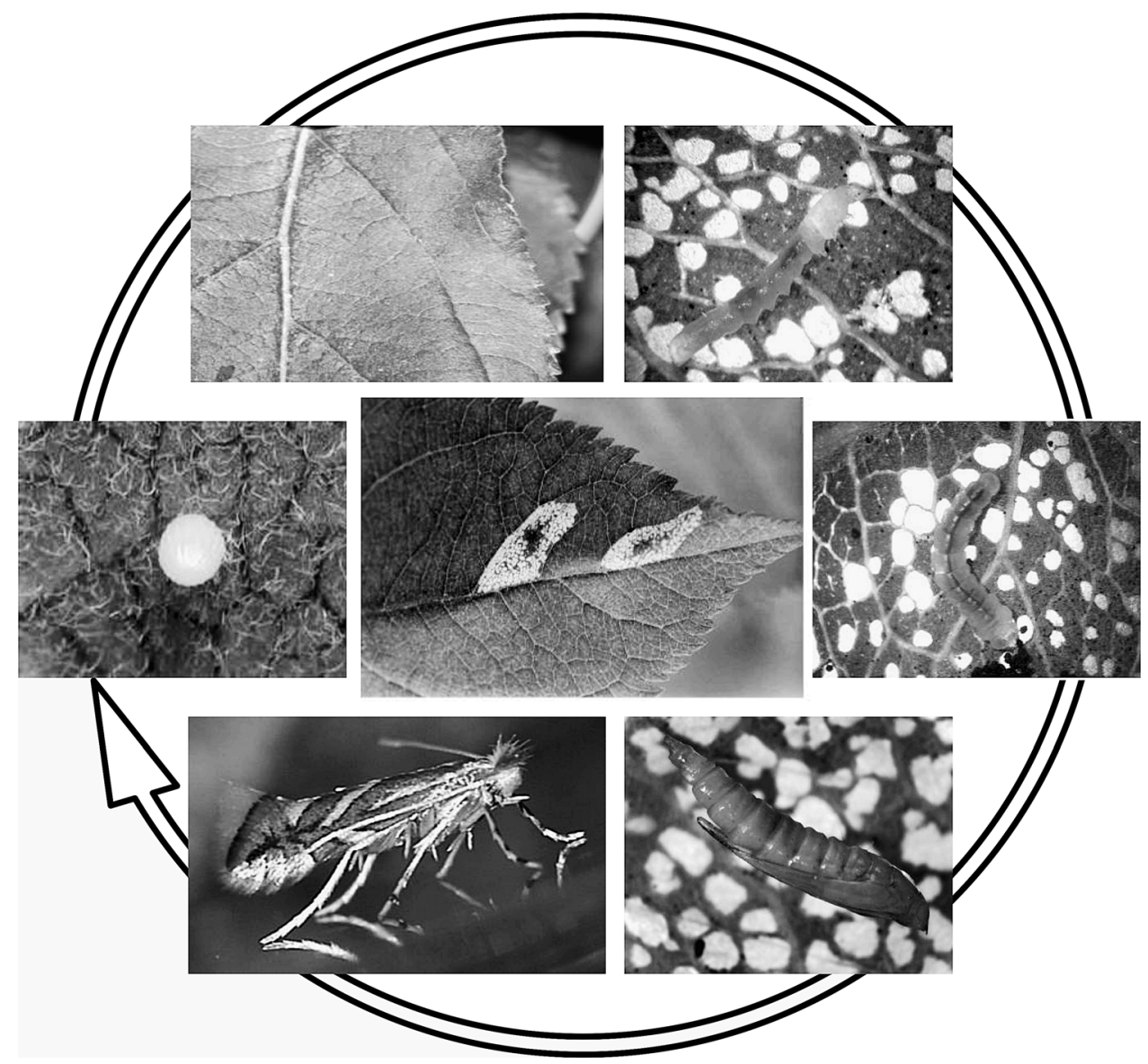

Plate 1. The life cycle of the leaf miner Phyllonorycter blancardella. The egg is deposited on the lower surface of an apple leaf. The first three larval stages (L1-3) are sap feeders and the damage is barely visible (leaf surface image). Then, the larva starts feeding on tissues at the fourth larval stage, creating feeding windows (white patches on the leaf surface after opening the mine). More feeding windows appear at the fifth larval stage, as the larva eats at a faster rate. Finally, the insect pupate within the mine, and the adult eventually emerges from its mine. The image in the center of the cycle shows two L5 mines at the apple leaf surface. Photo credits: S. Pincebourde.

feeding rate (Pincebourde et al. 2012), and population dynamics (Estay et al. 2011, 2014). These effects of daily fluctuating regimes are often well explained by the shape of the nonlinear reaction norm where the upper thermal limit is often closer to the optimum than the lower limit (Foray et al. 2014). Predicting the effect of microclimate variations on physiological rates, including developmental, ingestion, and assimilation rates, and ultimately on fitness and demographic performance, therefore requires the integration of daily fluctuations and reaction norms including physiological limits.

The biophysical model of microclimate temperature provides a good mechanistic understanding of the microclimate shift in a leaf mine across its inhabitant's life cycle. Under identical environmental conditions in full sunlight, the L5 mine of $P$. blancardella has microclimate temperatures up to $6^{\circ} \mathrm{C}$ above those experienced by the eggs at the leaf surface or by young L4 larvae. The microclimatic change in L5 mines and pupae is related to the amount of incoming radiation transmitted by the feeding windows (Pincebourde and Casas 2006b). The mine surface absorbs much more near-infrared radiation than do intact leaf tissues (Pincebourde and Casas 2006a). In addition, the response of stomata in the mine integument (i.e. closing above moderate radiation levels) amplifies the warming effect (Pincebourde et al. 2006). This microclimatic change put this leaf miner at greater risk in warming climates, compared to other insect species living in microhabitats that buffer environmental extremes (Saudreau et al. 2013). The feeding activity of this herbivore, therefore, drives directly the micro-environmental conditions that it experiences. Complex feedbacks between microclimate temperature and the insect responses are expected because the feeding dynamics may differ according to microclimate temperature. The leaf miner never eats more than $\sim 50 \%$ of the total mined area, supposedly to use the remaining tissues as a shield 
against parasitoid attacks (Djemai et al. 2000). Feeding more or less than this portion would increase or decrease the temperature within a mine, respectively, but it is not known whether this threshold shifts with environmental conditions.

Although leaf miners are particular in that they have an intimate relationship with plants by living inside them, we expect a strong coupling between microhabitat and thermal ecology across ontogeny in externally feeding insects as well. For example, the eggs and young larvae of the moth Manduca sexta are protected against heat in the desert by living at the leaf surface, whereas older caterpillars experience higher body temperatures by living out of the leaf boundary layer (Woods 2013). Thermal limits are not precisely known for all Manduca stages, but eggs are less tolerant than larvae by about $1-2^{\circ} \mathrm{C}$ (Casey 1976, Potter and Woods 2012). Interestingly, pupae have a lower tolerance threshold than eggs and larvae. The pupa, however, is found 5-30 $\mathrm{cm}$ deep into the soil, where the extreme environmental temperatures are buffered (Kingsolver et al. 2011). Each life stage is therefore specifically adapted to its microenvironment. This exemplifies the difficulty of laying out a general pattern on the role of ontogeny and aging in the evolution (or acclimation) of insect thermal tolerance (Bowler and Terblanche 2008). Various trends of thermal adaptation across ontogeny are expected according to microclimate shifts caused by changes in life histories occurring during growth.

Microclimate temperatures experienced during ontogeny remain unknown for a vast majority of organisms (Suggitt et al. 2011, Pincebourde and Woods 2012, Potter et al. 2013, Caillon et al. 2014). Data on microclimate shifts across ontogeny of ectotherms are even more sparse (Briscoe et al. 2012, Scheffers et al. 2013a, Woods 2013). Continuous measurements of microclimate temperatures at each locality within species distributions, and for each life stage, are logistically difficult. Alternatively, the known thermal limits could be used to infer the highest microclimatic temperatures that a species experiences during its development. Then, this inferred microclimate pattern reflects whether the microhabitat buffers (e.g., forest undercover; Scheffers et al. 2013b) or amplifies (e.g., leaf surfaces; Pincebourde and Woods 2012) the macroclimate temperature fluctuations (Fig. 1). This inverse approach was used recently to infer temperature patterns at local scales from the distribution and thermal needs of 872 plant species (Lenoir et al. 2013). This study revealed that the thermal variability within $1 \mathrm{~km}^{2}$ strongly buffers the amplitude of future global warming in Northern Europe. The inverse approach provides a pertinent alternative to extensive microclimate sampling for estimating microclimate temperatures and hence warming tolerance of ectotherms.

Warming tolerance would appear to be a straightforward metric that indicates the vulnerability of organisms to global warming (Deutsch et al. 2008, Diamond et al. 2012). We show, however, that accurately assessing the warming tolerance of terrestrial ectotherms involves more than coupling macroclimate temperatures to thermal limits. Rather, it involves understanding the microclimate temperatures experienced by ectotherms (Kearney 2013). Naïve warming tolerance, which ignores microclimates (Scheffers et al. 2013a), is likely to give erroneous predictions on distribution shifts at the warm boundary of species ranges, depending on the microhabitat characteristics (Fig. 1). For example, when microclimate reaches temperatures higher than macroclimate temperature, as it is the case for leaf miners, a species may disappear from a given geographic area even if its thermal limit remain well above macroclimate temperature. In this case, the habitat-specific warming tolerance gives more alarming forecasts than does the naïve warming tolerance in terms of species persistence. Conversely, species living in microhabitats that buffer extreme macroclimate temperatures can persist in a given geographic area despite macroclimate temperature that is already above their upper thermal limit. And here forecasts of species persistence are less alarming when based on the habitat-specific warming tolerance rather than on the naïve warming tolerance. We show that the naïve approach overestimated by at least $7^{\circ} \mathrm{C}$ (and up to $11^{\circ} \mathrm{C}$ ) the warming tolerance of the leaf miner (Fig. 7), a bias that is half of the naïve warming tolerance expected in general for insects at latitudes around $40^{\circ} \mathrm{N}$ (Deutsch et al. 2008). Fortunately, an extensive suite of biophysical models is available to simulate microclimate temperatures over the long term for major habitats (Kearney and Porter 2009), including soil layers (Kearney et al. 2014), leaf surfaces (Pincebourde et al. 2007), or fruit volumes (Saudreau et al. 2009). Knowing the life histories of each species, one could then use the adequate habitat model for each life stage to simulate the microclimate temperature experienced over the entire life of an organism. These approaches are among the most promising candidates to form the basis for predictive mechanistic models of climate change impacts that are needed to estimate shifts in distribution range and species persistence in the future.

\section{ACKNOWLedgments}

We thank Arthur Woods, two anonymous reviewers, and T. D. Williams for insightful comments on a previous version of the manuscript. We are grateful to Sophie Delaide for her help during some of the experimental procedures, and to the ESMB group at IRBI for fruitful discussions. This work was supported by ANR Blanc MicroCliMite (ANR-2010 BLAN1706-02).

\section{Literature Cited}

Addo-Bediako, A., S. L. Chown, and K. J. Gaston. 2000. Thermal tolerance, climatic variability and latitude. Proceedings of the Royal Society B 267:739-745. 
Angilletta, M. J. 2009. Thermal adaptation-A theoretical and empirical synthesis. Oxford University Press, Oxford, UK.

Araújo, M. B., F. Ferri-Yanes, F. Bozinovic, P. A. Marquet, F. Valladares, and S. L. Chown. 2013. Heat freezes niche evolution. Ecology Letters 16:1206-1219.

Bowler, K., and J. S. Terblanche. 2008. Insect thermal tolerance: what is the role of ontogeny, ageing and senescence? Biological Reviews 83:339-355.

Briscoe, N. J., W. P. Porter, P. Sunnucks, and M. R. Kearney. 2012. Stage-dependent physiological responses in a butterfly cause non-additive effects on phenology. Oikos 121:14641472.

Buckley, L. B., M. C. Urban, M. J. Angilletta, L. G. Crozier, L. J. Rissler, and M. W. Sears. 2010. Can mechanism inform species' distribution models? Ecology Letters 13:1041-1054.

Caillon, R., C. Suppo, J. Casas, H. A. Woods, and S. Pincebourde. 2014. Warming decreases thermal heterogeneity of leaf surfaces: Implications for behavioural thermoregulation by arthropods. Functional Ecology 28:1449-1458.

Campbell, G. S., and J. M. Norman. 1998. An introduction to environmental biophysics. Second edition. Springer Verlag, New York, New York, USA.

Casey, T. M. 1976. Activity patterns, body temperature, and thermal ecology in two desert caterpillars (Lepidoptera: Sphingidae). Ecology 57:485-497.

Crill, W. D., R. B. Huey, and G. W. Gilchrist. 1996. Within- and between-generation effects of temperature on the morphology and physiology of Drosophila melanogaster. Evolution 50: 1205-1218.

Denny, M., L. Hunt, L. Miller, and C. Harley. 2009. On the prediction of extreme ecological events. Ecological Monographs 79:397-421.

Deutsch, C. A., J. J. Tewksbury, R. B. Huey, K. S. Sheldon, C. K. Ghalambor, D. C. Haak, and P. R. Martin. 2008. Impacts of climate warming on terrestrial ectotherms across latitude. Proceedings of the National Academy of Sciences USA 105:6668-6672.

Diamond, S., D. Sorger, J. Hulcr, S. Pelini, I. Del Toro, C. Hirsch, E. Oberg, and R. R. Dunn. 2012. Who likes it hot? A global analysis of the climatic, ecological, and evolutionary determinants of warming tolerance in ants. Global Change Biology 18:448-456.

Djemai, I., R. Meyhöfer, and J. Casas. 2000. Geometrical games between a host and a parasitoid. American Naturalist 156:257-265.

Estay, S. A., S. Clavijo-Baquet, M. Lima, and F. Bozinovic. 2011. Beyond average: an experimental test of temperature variability on the population dynamics of Tribolium confusum. Population Ecology 53:53-58.

Estay, S. A., M. Lima, and F. Bozinovic. 2014. The role of temperature variability on insect performance and population dynamics in a warming world. Oikos 123:131-140.

Folguera, G., D. A. Bastias, J. Caers, J. M. Rojas, M. D. Piulachs, X. Bellés, and F. Bozinovic. 2011. An experimental test of the role of environmental temperature variability on ectotherm molecular, physiological and life-history traits: Implications for global warming. Comparative Biochemistry and Physiology A 159:242-246.

Foray, V., E. Desouhant, and P. Gibert. 2014. The impact of thermal fluctuations on reaction norms in specialist and generalist parasitic wasps. Functional Ecology 28:411-423.

Gates, D. M. 1980. Biophysical ecology. Springer-Verlag, New York, New York, USA.

Helmuth, B. 1998. Intertidal mussel microclimates: predicting the body temperature of a sessile invertebrate. Ecological Monographs 68:51-74.

Helmuth, B., B. R. Broitman, C. Blanchette, S. E. Gilman, P. Halpin, C. D. G. Harley, M. J. O'Donnell, G. E. Hofmann, B. Menge, and D. Strickland. 2006. Mosaic patterns of thermal stress in the rocky intertidal zone: implications for climate change. Ecological Monographs 76:461-479.
Helmuth, B., B. R. Broitman, L. Yamane, S. E. Gilman, K. Mach, K. A. S. Mislan, and M. W. Denny. 2010. Organismal climatology: analyzing environmental variability at scales relevant to physiological stress. Journal of Experimental Biology 213:995-1003.

Hoffmann, A. A., S. L. Chown, and S. Clusella-Trullas. 2013. Upper thermal limits in terrestrial ectotherms: how constrained they are? Functional Ecology 27:934-949.

Huey, R. B., M. R. Kearney, A. Krockenberger, J. A. M. Holtum, M. Jess, and S. E. Williams. 2012. Predicting organismal vulnerability to climate warming: incorporating the buffering roles of behaviour, physiology, and adaptation. Philosophical Transactions of the Royal Society B 367:16651679.

Huey, R. B., and J. G. Kingsolver. 1993. Evolution of resistance to high temperature in ectotherms. American Naturalist 142: S21-S46.

Jarvis, P. G. 1976. The interpretation of the variations in leaf water potential and stomatal conductance found in canopies in the field. Philosophical Transactions of the Royal Society B 273:593-610.

Kearney, M. R. 2013. Activity restriction and the mechanistic basis for extinctions under climate warming. Ecology Letters 16:1470-1479.

Kearney, M. R., N. J. Briscoe, D. J. Karoly, W. P. Porter, M. Norgate, and P. Sunnucks. 2010a. Early emergence in a butterfly causally linked to anthropogenic warming. Biology Letters 6:674-677.

Kearney, M., and W. P. Porter. 2009. Mechanistic niche modelling: combining physiological and spatial data to predict species' ranges. Ecology Letters 12:334-350.

Kearney, M. R., A. Shamakhy, R. Tingley, D. J. Karoly, A. A. Hoffmann, P. R. Briggs, and W. P. Porter. 2014. Microclimate modelling at macro scales: a test of a general microclimate model integrated with gridded continental-scale soil and weather data. Methods in Ecology and Evolution 5: 273-286.

Kearney, M. R., S. J. Simpson, D. Raubenheimer, and B. Helmuth. 2010b. Modelling the ecological niche from functional traits. Philosophical Transactions of the Royal Society B 365:3469-3483.

Kingsolver, J. G., S. E. Diamond, and L. B. Buckley. 2013. Heat stress and the fitness consequences of climate change for terrestrial ectotherms. Functional Ecology 27:1415-1423.

Kingsolver, J. G., G. J. Ragland, and S. E. Diamond. 2009. Evolution in the constant environment: thermal fluctuations and thermal sensitivity in laboratory and field populations of Manduca sexta. Evolution 63:537-541.

Kingsolver, J. G., H. A. Woods, L. B. Buckley, K. A. Potter, H. J. MacLean, and J. K. Higgins. 2011. Complex life cycles and the responses of insects to climate change. Integrative and Comparative Biology 51:719-732.

Lenoir, J., et al. 2013. Local temperatures inferred from plant communities suggest strong spatial buffering of climate warming across Northern Europe. Global Change Biology 19:1470-1481

Natori, Y., and W. P. Porter. 2007. Model of Japanese serow (Capricornis crispus) energetics predicts distribution on Honshu, Japan. Ecological Applications 17:1441-1459.

Nobel, P. S. 1999. Physicochemical and environmental plant physiology. Second edition. Academic Press, New York, New York, USA.

Nyamukondiwa, C., and J. Terblanche. 2010. Within-generation variation of critical thermal limits in adult Mediterranean and natal fruit flies Ceratitis capitata and Ceratitis rosa: thermal history affects short-term responses to temperature. Physiological Entomology 35:255-264.

Paaijmans, K. P., R. L. Heinig, R. A. Seliga, J. I. Blanford, S. Blanford, C. C. Murdock, and M. B. Thomas. 2013. Temperature variation makes ectotherms more sensitive to climate change. Global Change Biology 19:2373-2380. 
Pincebourde, S., and J. Casas. 2006a. Multitrophic biophysical budgets: thermal ecology of an intimate herbivore insectplant interaction. Ecological Monographs 76:175-194.

Pincebourde, S., and J. Casas. 2006b. Leaf miner-induced changes in leaf transmittance cause variations in insect respiration rates. Journal of Insect Physiology 52:194-201.

Pincebourde, S., E. Frak, H. Sinoquet, J. L. Régnard, and J. Casas. 2006. Herbivory mitigation through increased water use efficiency in a leaf mining moth-apple tree relationship. Plant Cell and Environment 29:2238-2247.

Pincebourde, S., E. Sanford, J. Casas, and B. Helmuth. 2012. Temporal coincidence of environmental stress events modulates predation rates. Ecology Letters 15:680-688.

Pincebourde, S., E. Sanford, and B. Helmuth. 2008. Body temperature during low tide alters the feeding performance of a top intertidal predator. Limnology and Oceanography 53: $1562-1573$.

Pincebourde, S., H. Sinoquet, D. Combes, and J. Casas. 2007. Regional climate modulates the canopy mosaic of favourable and risky microclimates for insects. Journal of Animal Ecology 76:424-438.

Pincebourde, S., and H. A. Woods. 2012. Climate uncertainty on leaf surfaces: the biophysics of leaf microclimates and their consequences for leaf-dwelling organisms. Functional Ecology 26:844-853.

Potter, K., G. Davidowitz, and H. A. Woods. 2011. Cross-stage consequences of egg temperature in the insect Manduca sexta. Functional Ecology 25:548-556.

Potter, K. A., and H. A. Woods. 2012. No evidence for the evolution of thermal or desiccation tolerance of eggs among populations of Manduca sexta. Functional Ecology 26:112122.

Potter, K. A., H. A. Woods, and S. Pincebourde. 2013. Microclimatic challenges in global change biology. Global Change Biology 19:2932-2939.

Rezende, E. L., L. E. Castaneda, and M. Santos. 2014. Tolerance landscapes in thermal ecology. Functional Ecology 28:799-809.

Rezende, E. L., and M. Santos. 2012. Comment on 'Ecologically relevant measures of tolerance to potentially lethal temperatures'. Journal of Experimental Biology 215:702-703.

Rezende, E. L., M. Tejedo, and M. Santos. 2011. Estimating the adaptive potential of critical thermal limits: methodological problems and evolutionary implications. Functional Ecology 25:111-121.

Ribeiro, P. L., A. Camacho, and C. A. Navas. 2012. Considerations for assessing maximum critical temperatures in small ectothermic animals: insights from leaf-cutting ants. PLoS ONE 7:e32083.

Roberts, S. P., and M. E. Feder. 1999. Natural hyperthermia and expression of the heat shock protein Hsp70 affect developmental abnormalities in Drosophila melanogaster. Oecologia 121:323-329.
Santos, M., L. E. Castaneda, and E. L. Rezende. 2011. Making sense of heat tolerance estimates in ectotherms: lessons from Drosophila. Functional Ecology 25:1169-1180.

Saudreau, M., A. Marquier, B. Adam, P. Monney, and H. Sinoquet. 2009. Experimental study of fruit temperature dynamics within apple tree crowns. Agricultural and Forest Meteorology 149:362-372.

Saudreau, M., S. Pincebourde, M. Dassot, B. Adam, H. D. Loxdale, and D. G. Biron. 2013. On the canopy structure manipulation to buffer climate change effects on insect herbivore development. Trees-Structure and Function 27: 239-248.

Scheffers, B. R., R. M. Brunner, S. D. Ramirez, L. P. Shoo, A. Diesmos, and S. E. Williams. 2013a. Thermal buffering of microhabitats is a critical factor mediating warming vulnerability of frogs in the Philippine biodiversity hotspot. Biotropica 45:628-635.

Scheffers, B. R., D. P. Edwards, A. Diesmos, S. E. Williams, and T. A. Evans. 2013b. Microhabitats reduce animal's exposure to climate extremes. Global Change Biology 20: 495-503.

Stillman, J. H., and G. N. Somero. 2000. A comparative analysis of the upper thermal tolerance limits of eastern Pacific porcelain crabs, genus Petrolisthes: influences of latitude, vertical zonation, acclimation, and phylogeny. Physiological and Biochemical Zoology 73:200-208.

Suggitt, A. J., P. K. Gillingham, J. K. Hill, B. Huntley, W. E. Kunin, D. B. Roy, and C. D. Thomas. 2011. Habitat microclimates drive fine-scale variation in extreme temperatures. Oikos 120:1-8.

Sunday, J., A. E. Bates, and N. K. Dulvy. 2011. Global analysis of thermal tolerance and latitude in ectotherms. Proceedings of the Royal Society B 278:1823-1830.

Sunday, J. M., A. E. Bates, and N. K. Dulvy. 2012. Thermal tolerance and the global redistribution of animals. Nature Climate Change 2:686-690.

Sunday, J. M., A. E. Bates, M. R. Kearney, R. K. Colwell, N. K. Dulvy, J. T. Longino, and R. B. Huey. 2014. Thermalsafety margins and the necessity of thermoregulatory behavior across latitude and elevation. Proceedings of the National Academy of Sciences USA 111:5610-5615.

Terblanche, J., A. Hoffmann, K. Mitchell, L. Rako, P. le Roux, and S. Chown. 2011. Ecologically relevant measures of tolerance to potentially lethal temperatures. Journal of Experimental Biology 214:3713-3725.

Tewksbury, J. J., R. B. Huey, and C. A. Deutsch. 2008. Putting the heat on tropical animals. Science 320:1296-1297.

Williams, S. E., L. Shoo, J. Isaac, A. A. Hoffmann, and G. Langham. 2008. Towards an integrated framework for assessing the vulnerability of species to climate change. PLoS Biology 6:2621-2626.

Woods, H. A. 2013. Ontogenetic changes in the body temperature of an insect herbivore. Functional Ecology 27: $1322-1331$

\section{Supplemental Material}

\section{Ecological Archives}

The Appendix is available online: http://dx.doi.org/10.1890/14-0744.1.sm 